\title{
THE STRONG REES PROPERTY OF POWERS OF THE MAXIMAL IDEAL AND TAKAHASHI-DAO'S QUESTION
}

\author{
TONY J.PUTHENPURAKAL, KEI-ICHI WATANABE, KEN-ICHI YOSHIDA \\ Dedicated to Craig Huneke on the occasion of his 65th Birthday.
}

\begin{abstract}
In this paper, we introduce the notion of the strong Rees property (SRP) for $\mathfrak{m}$-primary ideals of a Noetherian local ring and prove that any power of the maximal ideal $\mathfrak{m}$ has its property if the associated graded ring $G$ of $\mathfrak{m}$ satisfies $\operatorname{depth} G \geq 2$. As its application, we characterize two-dimensional excellent normal local domains so that $\mathfrak{m}$ is a $p_{g}$-ideal.

Finally we ask what $\mathfrak{m}$-primary ideals have SRP and state a conjecture which characterizes the case when $\mathfrak{m}^{n}$ are the only ideals which have SRP.
\end{abstract}

\section{INTRODUCTION}

Let $(A, \mathfrak{m})$ be a Noetherian local ring with $d=\operatorname{dim} A \geq 1$ and $I$ an $\mathfrak{m}$-primary ideal of $A$. The notion of $\mathfrak{m}$-full ideals was introduced by D. Rees and J. Watanabe ([22]) and they proved the "Rees property" for $\mathfrak{m}$-full ideals, namely, if $I$ is $\mathfrak{m}$-full ideal and $J$ is an ideal containing $I$, then $\mu(J) \leq \mu(I)$, where $\mu(I)=\ell_{A}(I / \mathfrak{m} I)$ is the minimal number of generators of $I$. Also, they proved that integrally closed ideals are $\mathfrak{m}$-full if $A$ is normal.

Suppose $\operatorname{depth} A>0$. Then $\widetilde{\mathfrak{m}^{n}}$, the Ratliff-Rush closure of $\mathfrak{m}^{n}$, is $\mathfrak{m}$-full ([1, Proposition 2.2]). Thus $\mathfrak{m}^{n}$ is $\mathfrak{m}$-full for sufficiently large $n$.

Sometimes we need stronger property for $\mu(I)$ and we will call it "Strong Rees property" (SRP for short).

Definition (Strong Rees Property). Let $I$ be an m-primary ideal of $A$. Then we say that $I$ satisfies the strong Rees property if for every ideal $J \supsetneq I$, we have $\mu(J)<\mu(I)$.

So it will be natural to ask the following questions.

Question 1.1. Let $(A, \mathfrak{m})$ be a normal local ring. Then

(1) Does $\mathfrak{m}^{n}$ has the strong Rees property for every $n \geq 1$ ?

(2) If I has the strong Rees property, then is $I=\mathfrak{m}^{n}$ for some $n \geq 1$ ?

Date: September 24, 2018.

2000 Mathematics Subject Classification. Primary 13A30; Secondary 13H15, 13B22, 14B05. 
Actually, both questions are not true in general. But we can show that (1) in the Question 1.1 holds under suitable mild condition. Also, we will give an example of two-dimensional normal local rings where $\mathfrak{m}^{2}$ does not satisfy the strong Rees property.

As for (2) of Question 1.1, we will discuss it in Section 6.

Assume that $I$ is an m-primary ideal. Then the multiplicity (resp. the minimal number of system of generators) of $I$ is denoted by $e(I)$ (resp. $\mu(I)$ ). The Loewy length $\ell \ell(I)$ is defined by

$$
\ell \ell(I)=\min \left\{r \in \mathbb{Z}_{\geq 0} \mid \mathfrak{m}^{r} \subset I\right\}
$$

Notice that the notion of Loewy length of an Artinian ring measures the nilpotency of the maximal ideal. It is natural to ask if $e(I)$ is bounded by the product of $\mu(I)$ and $\ell \ell(I)$ after adjusting some error terms.

The origin of this work was a discussion of second and third authors with Hailong Dao. He presented an inequality between $\mu(I)$, the multiplicity $e(I)$ of $I$ and Loewy length $\ell \ell(I)$.

Question 5.1 ([2]). Let $(A, \mathfrak{m})$ be a $d$-dimensional Cohen-Macaulay local ring. Let $I \subset A$ be an $\mathfrak{m}$-primary (integrally closed) ideal. When does the inequality

$$
(d-1) !(\mu(I)-d+1) \cdot \ell \ell(I) \geq e(I)
$$

hold true?

Dao and Smirnov 2] proved that the Question 5.1 holds true if $A$ is a twodimensional analytically unramified and the maximal ideal $\mathfrak{m}$ is a $p_{g}$-ideal (or, equivalently, the Rees algebra $\mathcal{R}(\mathfrak{m})$ is normal and Cohen-Macaulay).

We are interested in the converse of the Question 5.1 in the case of $d=2$. Here, since $e(I)$ does not change after taking integral closure, it is natural to assume that $I$ is integrally closed. Namely, our Question is

Question 1.2. Assume that $(A, \mathfrak{m})$ is a two-dimensional excellent normal local domain. If an inequality

$$
(\mu(I)-1) \cdot \ell \ell(I) \geq e(I)
$$

holds for any $\mathfrak{m}$-primary integrally closed ideal $I$, then is $\mathfrak{m}$ a $p_{g}$-ideal?

It turns out that this question is related to the "strong Rees property" of powers of the maximal ideal. The main result in this paper is the following theorem.

Theorem 3.2. Let $(A, \mathfrak{m})$ be a Noetherian local ring. Assume that $\operatorname{depth} A \geq 2$ and $H_{\mathfrak{M}}^{1}(G)$ has finite length, where $G=G(\mathfrak{m})=\oplus_{n \geq 0} \mathfrak{m}^{n} / \mathfrak{m}^{n+1}$. If $\mathfrak{m}^{\ell}$ is RatliffRush closed, then $\mathfrak{m}^{\ell}$ has the strong Rees property.

By Remark 2.5, any power $\mathfrak{m}^{\ell}$ is Ratliff-Rush closed whenever $\operatorname{depth} G \geq 1$. Hence we have the following corollary. 
Corollary 3.5. If $\operatorname{depth} G \geq 2$, then $\mathfrak{m}^{\ell}$ has the strong Rees property for every $\ell \geq 1$.

In general, we cannot relax the assumption that $\operatorname{depth} G \geq 2$ even if $A$ is normal. See Section 4 for more details.

In Section 5, as an application of the theorem above, we prove the above question has an affirmative answer.

\section{Theorem 5.2 ,}

Let $(A, \mathfrak{m})$ be a two-dimensional excellent normal local domain containing an algebraically closed field. Then the following conditions are equivalent:

(1) $\mathfrak{m}$ is a $p_{g}$-ideal.

(2) For every m-primary integrally closed ideal $I$,

$$
(\mu(I)-1) \cdot \ell \ell_{A}(I) \geq e(I)
$$

holds true.

(3) For any power $I=\mathfrak{m}^{\ell}$ of $\mathfrak{m}$, the inequality $(*)$ holds true.

After proving this theorem, Dao and Smirnov informed us that they proved the same theorem independently.

\section{Preliminaries}

Throughout this paper, let $(A, \mathfrak{m})$ be a Noetherian local $\operatorname{ring}$ with $d=\operatorname{dim} A \geq 1$, and let $I, J \subset A$ be ideals of positive height. We recall the notion which we will need later.

\section{1. $\mathfrak{m}$-full ideals.}

Definition 2.1 ( $\mathfrak{m}$-full, Rees property[22]). An ideal $I$ is called $\mathfrak{m}$-full if there exists an element $x \in \mathfrak{m}$ so that $\mathfrak{m} I: x=I$.

An ideal $I$ is said to have the Rees property if $\mu(J) \leq \mu(I)$ for any ideal $J$ containing $I$.

Proposition 2.2 (See [22, Theorem 3]). Any $\mathfrak{m}$-primary $\mathfrak{m}$-full ideal has the Rees property.

Proof. See the proof of [3, Lemma 2.2].

The following result is due to Rees in the case of normal integral domains.

Proposition 2.3 (See [22, Theorem 5]). Any integrally closed ideal of positive height is $\mathfrak{m}$-full.

Proof. See the proof of Theorem [3, Theorem 2.4]. 
Definition 2.4 (Ratliff-Rush closure [17). The Ratliff-Rush closure of $I$ is defined by

$$
\widetilde{I}=\bigcup_{n \geq 0} I^{n+1}: I^{n}
$$

The ideal $I$ is called Ratliff-Rush closed if $\widetilde{I}=I$.

Remark 2.5. Note that $I \subset \widetilde{I} \subset \bar{I}$, where $\bar{I}$ is the integrally closure of $I$. If we put $G=G(\mathfrak{m})=\bigoplus_{n \geq 0} \mathfrak{m}^{n} / \mathfrak{m}^{n+1}$, then

$$
\left.H_{\mathfrak{M}}^{0}(G)=\bigoplus_{n \geq 0} \widetilde{\left(\mathfrak{m}^{n+1}\right.} \cap \mathfrak{m}^{n}\right) / \mathfrak{m}^{n+1} .
$$

Lemma 2.6. Assume that for some $z \in \mathfrak{m}^{n-1} \backslash \mathfrak{m}^{n}$ we have $z \mathfrak{m} \subset \mathfrak{m}^{n-1}$. Then, putting $I=(x)+\mathfrak{m}^{n}, \mu(I)>\mu\left(\mathfrak{m}^{n}\right)$, hence $\mathfrak{m}^{n}$ does not have Rees property.

Proof. Let $\left\{y_{1}, \ldots, y_{\mu}\right\}$ be a minimal set of generators of $\mathfrak{m}^{n}$. Then we can see that $\left\{z, y_{1}, \ldots, y_{\mu}\right\}$ is a minimal set of generators of $I$. Hence $\mu(I)=\mu\left(\mathfrak{m}^{n}\right)+1$.

Any $\mathfrak{m}$-full ideal has the Rees property. However, in order to prove our theorem, we need stronger inequalities.

Recall the definition of the strong Rees property.

Definition 2.7. An m-primary ideal $I$ is said to have the strong Rees property (SRP for short) if $\mu(J)<\mu(I)$ holds true for every ideal $J$ with $I \subsetneq J$.

We can show the existence of $\mathfrak{m}$-primary ideals with SRP by the following Lemma.

Lemma 2.8. Let $(A, \mathfrak{m})$ be a Noetherian local ring. Fix an $\mathfrak{m}$-primary ideal I with $\mu(I)=n$.

(1) Any maximal element of the set of $\mathfrak{m}$-primary ideals

$$
\mathcal{I}=\{J \subset A \mid J \supset I \text { and } \mu(J) \geq n\}
$$

has the strong Rees property.

(2) If, moreover, I is $\mathfrak{m}$-full, then there is an ideal $I^{\prime} \supset I$ with the strong Rees property and $\mu\left(I^{\prime}\right)=\mu(I)$.

Proof. (1) is obvious by the definition of $\mathcal{I}$.

(2) If $I^{\prime} \supset I$ has SRP and $\mu\left(I^{\prime}\right) \geq \mu(I)$, we should have $\mu\left(I^{\prime}\right)=\mu(I)$ since $I$ is $\mathfrak{m}$-full.

The next example gives a motivation for us to study the strong Rees property of powers of the maximal ideal. See also [22, Theorem 5] and Section 6 .

Example 2.9. Assume that $A$ is a two-dimensional regular local ring. Then for any $\mathfrak{m}$-primary ideal $I$, the following conditions are equivalent.

(1) $I$ has the strong Rees property. 
(2) $I=\mathfrak{m}^{n}$ for some integer $n \geq 1$.

Indeed, assume that $I$ has the strong Rees property. If we put $n=\operatorname{ord}(I)=$ $\max \left\{n \in \mathbb{Z} \mid I \subset \mathfrak{m}^{n}\right\}$, then we can take an element $f \in I$ so that $\operatorname{ord}(f)=$ $n$. Then since $I /(f)$ is an $\mathfrak{m} /(f)$-primary ideal of $A /(f)$, we have $\mu(I /(f)) \leq$ $n=e(\mathfrak{m} /(f))$ because $A /(f)$ is a one-dimensional Cohen-Macaulay local ring. In particular, $\mu(I) \leq n+1=\mu\left(\mathfrak{m}^{n}\right)$. If $I \neq \mathfrak{m}^{n}$, then $n+1=\mu\left(\mathfrak{m}^{n}\right)<\mu(I)$ by the assumption (1). But this is a contradiction.

Conversely, if $I \supsetneq \mathfrak{m}^{n}$, then $\operatorname{ord}(I)<n$ and $\mu(I) \leq \operatorname{ord}(I)+1<n+1=\mu\left(\mathfrak{m}^{n}\right)$.

2.2. $p_{g}$-ideals. In what follows, let $(A, \mathfrak{m})$ be a two-dimensional excellent normal local domain containing an algebraically closed field $k=A / \mathfrak{m}$. Let $f: X \rightarrow \operatorname{Spec} A$ be a resolution of singularities. Then $p_{g}(A)=\operatorname{dim}_{k} H^{1}\left(\mathcal{O}_{X}\right)$ is called the geometric genus of $A$. Note that it does not depend on the choice of resolution of singularities.

Let $I \subset A$ be an m-primary integrally closed ideal. Let $f: X \rightarrow \operatorname{Spec} A$ be a resolution of singularities on which $I$ is represented, that is, $I \mathcal{O}_{X}$ is invertible and $I \mathcal{O}_{X}=\mathcal{O}_{X}(-Z)$ for some anti-nef cycle $Z$ on $X$.

Okuma and the last two authors 13 proved that $\operatorname{dim}_{k} H^{0}\left(\mathcal{O}_{X}(-Z)\right) \leq p_{g}(A)$ holds true if $H^{0}\left(\mathcal{O}_{X}(-Z)\right)$ has no fixed component.

Definition 2.10 (See [13]). An anti-nef cycle $Z$ is a $p_{g}$-cycle if $\mathcal{O}_{X}(-Z)$ is generated and $\operatorname{dim}_{k} H^{0}\left(\mathcal{O}_{X}(-Z)\right)=p_{g}(A)$. An m-primary integrally closed ideal $I$ is called a $p_{g}$-ideal if $I$ is represented as $I=H^{0}\left(\mathcal{O}_{X}(-Z)\right)$ by a $p_{g}$-cycle $Z$.

The following theorem gives a characterization of $p_{g}$-ideals in terms of Rees algebras.

Proposition 2.11 (See 14). Let $(A, \mathfrak{m})$ be a two-dimensional excellent normal local domain containing an algebraically closed field. Let $I \subset A$ be an $\mathfrak{m}$-primary ideal. Then the following conditions are equivalent:

(1) $I$ is a $p_{g}$-ideal.

(2) $\overline{I^{n}}=I^{n}$ for every $n \geq 1$ and $I^{2}=Q I$ for some minimal reduction $Q \subset I$.

(3) The Rees algebra $\mathcal{R}(I)=A[I t](\subset A[t])$ is a Cohen-Macaulay normal domain, where $t$ is an indeterminate over $A$.

For instance, any integrally closed $\mathfrak{m}$-primary ideal in a two-dimensional rational singularity (i.e. $p_{g}(A)=0$ ) is a $p_{g}$-ideal. On the other hand, any two-dimensional excellent normal local domain containing algebraically closed field has a $p_{g}$-ideal; see 13,14 . 


\section{Strong Rees Property of powers of the maximal ideal}

In what follows, let $(A, \mathfrak{m})$ be a Noetherian local ring and set $\mathcal{R}=\mathcal{R}(\mathfrak{m})$ and $G=G(\mathfrak{m})$ and $\mathfrak{M}=\mathfrak{m} \mathcal{R}+\mathcal{R}_{+}$. The main purpose of this section is to consider the following question.

Question 3.1. Assume that I is Ratliff-Rush closed. When does I have the strong Rees property?

As an answer, we show that some powers of the maximal ideal $\mathfrak{m}$ have the strong Rees property if $\operatorname{depth} G \geq 2$; see Corollary 3.5. More generally, we can show the following theorem.

Theorem 3.2 (Strong Rees Property). Assume that depth $A \geq 2$ and $H_{\mathfrak{M}}^{1}(G)$ has finite length. If $\mathfrak{m}^{\ell}$ is Ratliff-Rush closed, then $\mathfrak{m}^{\ell}$ has the strong Rees property.

We first need to prove the following lemma.

Lemma 3.3. Suppose that for some $x \in \mathfrak{m}, \mathfrak{m}^{\ell+1}: x=\mathfrak{m}^{\ell}$. Then for every ideal $J$ with $J \supset \mathfrak{m}^{\ell}, \mu(J) \leq \mu\left(\mathfrak{m}^{\ell}\right)$ is always satisfied, and the following conditions are equivalent:

(1) $\mu(J)=\mu\left(\mathfrak{m}^{\ell}\right)$.

(2) $\mathfrak{m} J=x J+\mathfrak{m}^{\ell+1}$.

When this is the case, if we put $C=J \mathcal{R} / \mathfrak{m}^{\ell} \mathcal{R}, C /(x t) C=(C /(x t) C)_{0}=J / \mathfrak{m}^{\ell}$. In particular, $\operatorname{dim} C \leq 1$.

Proof. Consider the following two short exact sequences:

$$
\begin{aligned}
0 & \rightarrow \frac{\mathfrak{m}^{\ell} \cap \mathfrak{m} J}{\mathfrak{m}^{\ell+1}} \rightarrow \frac{\mathfrak{m}^{\ell}}{\mathfrak{m}^{\ell+1}} \rightarrow \frac{\mathfrak{m}^{\ell}}{\mathfrak{m}^{\ell} \cap \mathfrak{m} J} \rightarrow 0 . \\
0 & \rightarrow \frac{\mathfrak{m}^{\ell}+\mathfrak{m} J}{\mathfrak{m} J} \rightarrow \frac{J}{\mathfrak{m} J} \rightarrow \frac{J}{\mathfrak{m}^{\ell}+\mathfrak{m} J} \rightarrow 0 .
\end{aligned}
$$

Since $\mathfrak{m}^{\ell} /\left(\mathfrak{m}^{\ell} \cap \mathfrak{m} J\right) \cong\left(\mathfrak{m}^{\ell}+\mathfrak{m} J\right) / \mathfrak{m} J$, combining two exact sequence implies

$$
0 \rightarrow \frac{\mathfrak{m}^{\ell} \cap \mathfrak{m} J}{\mathfrak{m}^{\ell+1}} \rightarrow \frac{\mathfrak{m}^{\ell}}{\mathfrak{m}^{\ell+1}} \rightarrow \frac{J}{\mathfrak{m} J} \rightarrow \frac{J}{\mathfrak{m}^{\ell}+\mathfrak{m} J} \rightarrow 0 .
$$

It follows that

$$
\ell_{A}\left(\mathfrak{m}^{\ell} \cap \mathfrak{m} J / \mathfrak{m}^{\ell+1}\right)=\left\{\mu\left(\mathfrak{m}^{\ell}\right)-\mu(J)\right\}+\ell_{A}\left(J / \mathfrak{m}^{\ell}+\mathfrak{m} J\right) .
$$

Furthermore, since $\mathfrak{m} J /\left(\mathfrak{m}^{\ell} \cap \mathfrak{m} J\right) \cong\left(\mathfrak{m}^{\ell}+\mathfrak{m} J\right) / \mathfrak{m}^{\ell}$, we get

$$
\ell_{A}\left(\mathfrak{m} J / \mathfrak{m}^{\ell+1}\right)=\ell_{A}\left(J / \mathfrak{m}^{\ell}\right)+\left\{\mu\left(\mathfrak{m}^{\ell}\right)-\mu(J)\right\}
$$

We now consider an $\mathcal{R}$-module $C=J \mathcal{R} / \mathfrak{m}^{\ell} \mathcal{R}$. The assumption that $\mathfrak{m}^{\ell+1}: x=$ $\mathfrak{m}^{\ell}$ implies that the multiplication map

$$
\cdot x: C_{0}=J / \mathfrak{m}^{\ell} \rightarrow C_{1}=\mathfrak{m} J / \mathfrak{m}^{\ell+1}
$$


is injective. Hence $\mu\left(\mathfrak{m}^{\ell}\right)-\mu(J)=\ell_{A}\left(C_{1}\right)-\ell_{A}\left(C_{0}\right) \geq 0$. Moreover, equality holds true if and only if the multiplication map by $x$ is isomorphism, which means that $\mathfrak{m} J=x J+\mathfrak{m}^{\ell+1}$.

When this is the case, we have

$$
\mathfrak{m}^{n+1} J=x \mathfrak{m}^{n} J+\mathfrak{m}^{n+\ell+1}
$$

for every $n \geq 1$. The last assertion immediately follows from this.

Lemma 3.4. Let $J \subset A$ be an ideal with $J \supsetneq \mathfrak{m}^{\ell}$, where $\ell \geq 1$. Put $C=J \mathcal{R} / \mathfrak{m}^{\ell} \mathcal{R}$. Assume that $\operatorname{depth} A \geq 2$, and $H_{\mathfrak{M}}^{1}(G)$ has finite length. If $\mathfrak{m}^{\ell}$ is Ratliff-Rush closed, then

(1) $H_{\mathfrak{M}}^{i}(C)$ has finite length for $i=0,1$.

(2) $\left[H_{\mathfrak{M}}^{0}(C)\right]_{0}=0$.

Proof. First we define an $\mathcal{R}$-module $L(-1)$ as follows:

$$
0 \rightarrow \mathcal{R} \rightarrow A[t] \rightarrow L(-1) \rightarrow 0(\mathrm{ex})
$$

that is, $L(-1)=\bigoplus_{n \geq 0}\left(A / \mathfrak{m}^{n}\right) t^{n}$.

Claim 1. $H_{\mathfrak{M}}^{i}(L(-1))$ has finite length for $i=0,1$.

By [15, Proposition 4.7] we have

$$
H_{\mathfrak{M}}^{0}(L(-1))=\bigoplus_{n \geq 0} \frac{\widetilde{\mathfrak{m}^{n}}}{\mathfrak{m}^{n}}=H_{\mathfrak{M}}^{0}(G),
$$

which has finite length and then it is proved in [16, Theorem 6.2] that $H_{\mathfrak{M}}^{1}(L(-1))$ has finite length, if and only if $H_{\mathfrak{M}}^{1}(G)$ has finite length. For instance, $\operatorname{depth} G \geq 2$, then $H_{\mathfrak{M}}^{i}(L(-1))=0$ for each $i=0,1$.

Secondly, we define $D=L(-1)_{\geq \ell}(\ell)=\bigoplus_{n \geq 0}\left(A / \mathfrak{m}^{\ell+n}\right) t^{n}$.

Claim 2. $\left[H_{\mathfrak{M}}^{0}(D)\right]_{0}=0$ and $H_{\mathfrak{M}}^{1}(D)$ has finite length.

By definition, we have

$$
0 \rightarrow D(-\ell) \rightarrow L(-1) \rightarrow W \rightarrow 0,
$$

where $W$ is an $\mathcal{R}$-module of finite length. Then since in the exact sequence

$$
W=H_{\mathfrak{M}}^{0}(W) \rightarrow H_{\mathfrak{M}}^{1}(D(-\ell)) \rightarrow H_{\mathfrak{M}}^{1}(L(-1))
$$

the modules of the both sides have finite length, so does $H_{\mathfrak{M}}^{1}(D(-\ell))$. Moreover, as $H_{\mathfrak{M}}^{0}\left(D(-\ell) \subset \subset H_{\mathfrak{M}}^{0}(L(-1)), H_{\mathfrak{M}}^{0}(D(-\ell))\right.$ is also of finite length.

The first assertion follows from the fact $\left[H_{\mathfrak{M}}^{0}(D)\right]_{0} \subset \widetilde{\mathfrak{m}^{\ell}} / \mathfrak{m}^{\ell}$ and our assumption.

Thirdly, we define an $\mathcal{R}$-module $V=\bigoplus_{n \geq 0}\left(A / \mathfrak{m}^{n} J\right) t^{n}$ as follows:

$$
0 \rightarrow J \mathcal{R} \rightarrow A[t] \rightarrow V \rightarrow 0(\mathrm{ex})
$$


By definition of $V$, we have

$$
0=H_{\mathfrak{M}}^{0}(A[t]) \rightarrow H_{\mathfrak{M}}^{0}(V) \rightarrow H_{\mathfrak{M}}^{1}(J \mathcal{R}) \rightarrow H_{\mathfrak{M}}^{1}(A[t])=0
$$

where two vanishing follows from the fact that $\operatorname{depth} A \geq 2$. It follows that $\left[H_{\mathfrak{M}}^{0}(V)\right]_{n} \cong\left[H_{\mathfrak{M}}^{1}(J \mathcal{R})\right]_{n}=0$ for large enough $n$. On the other hand, as $H_{\mathfrak{M}}^{0}(V) \subset$ $V,\left[H_{\mathfrak{M}}^{0}(V)\right]_{n}=0$ for each $n \leq-1$. Thus $H_{\mathfrak{M}}^{0}(V)$ has finite length.

Claim 3. $\left[H_{\mathfrak{M}}^{0}(C)\right]_{0}=0$ and $H_{\mathfrak{M}}^{1}(C)$ has finite length.

One can easily obtain the following exact sequence:

$$
0 \rightarrow C \rightarrow D \rightarrow V \rightarrow 0 \text { (ex). }
$$

Hence

$$
\begin{aligned}
0 & \rightarrow H_{\mathfrak{M}}^{0}(C) \rightarrow H_{\mathfrak{M}}^{0}(D) \rightarrow H_{\mathfrak{M}}^{0}(V) \\
& \rightarrow H_{\mathfrak{M}}^{1}(C) \rightarrow H_{\mathfrak{M}}^{1}(D) \rightarrow \cdots
\end{aligned}
$$

As $\left[H_{\mathfrak{M}}^{0}(D)\right]_{0}=0$ by Claim 2 , we get $\left[H_{\mathfrak{M}}^{0}(C)\right]_{0}=0$. Moreover, in the sequence

$$
H_{\mathfrak{M}}^{0}(D) \rightarrow H_{\mathfrak{M}}^{0}(V) \rightarrow H_{\mathfrak{M}}^{1}(C) \rightarrow H_{\mathfrak{M}}^{1}(D)
$$

the both sides of $H_{\mathfrak{M}}^{1}(C)$ have finite length. Hence so does $H_{\mathfrak{M}}^{1}(C)$.

Proof of Theorem 3.2, Choose an $\mathfrak{m}$-superficial element $x \in \mathfrak{m} \backslash \mathfrak{m}^{2}$. By assumption, we have

$$
\mathfrak{m}^{\ell} \subset \mathfrak{m}^{\ell+1}: x \subset \widetilde{\mathfrak{m}^{\ell}}=\mathfrak{m}^{\ell} .
$$

Then $\mathfrak{m}^{\ell+1}: x=\mathfrak{m}^{\ell}$ In particular, this means $\mathfrak{m}^{\ell}$ is $\mathfrak{m}$-full.

Let $J$ be an ideal with $J \supsetneq \mathfrak{m}^{\ell}$. By Lemma 3.3. $\mu(J) \leq \mu\left(\mathfrak{m}^{\ell}\right)$. We want to show that this inequality is strict. Now suppose that equality holds true: $\mu(J)=\mu\left(\mathfrak{m}^{\ell}\right)$. Put $C=J \mathcal{R} / \mathfrak{m}^{\ell} \mathcal{R}$. In Lemma 3.3, we showed that $C /(x t) C$ has finite length. Hence we have $\operatorname{dim} C \leq 1$.

On the other hand, by Lemma $\left[3.4\right.$, we have $\left[H_{\mathfrak{M}}^{0}(C)\right]_{0}=0$ and $H_{\mathfrak{M}}^{1}(C)$ has finite length. If $\operatorname{dim} C=0$, then $0 \neq J / \mathfrak{m}^{\ell}=[C]_{0}=\left[H_{\mathfrak{M}}^{0}(C)\right]_{0}=0$. This is a contradiction. Hence $\operatorname{dim} C=1$. Then $H_{\mathfrak{M}}^{1}(C)$ is not finitely generated. This contradicts the fact that $H_{\mathfrak{M}}^{1}(C)$ has finite length. Therefore we conclude that $\mu(J)<\mu\left(\mathfrak{m}^{\ell}\right)$, as required.

In the case where $\operatorname{depth} G \geq 2$, then all powers of the maximal ideal have the strong Rees property.

Corollary 3.5. If $\operatorname{depth} G \geq 2$, then $\mathfrak{m}^{\ell}$ has the strong Rees property for every $\ell \geq 1$.

Proof. Assume Theorem 3.2 holds true. If depth $G \geq 2$, then $H_{\mathfrak{M}}^{1}(G)=0$ and $\operatorname{depth} A \geq 2$. Hence the $\operatorname{ring} A$ satisfies the assumption on Theorem 3.2 . 
Corollary 3.6. Let $(A, \mathfrak{m})$ be a Cohen-Macaulay local ring with $d=\operatorname{dim} A \geq 2$. If $G=G(\mathfrak{m})$ is Cohen-Macaulay, then $\mathfrak{m}^{\ell}$ has the strong Rees property for each $\ell \geq 1$.

Corollary 3.7. If depth $A \geq 2$ and $\mathfrak{m}$ is a normal ideal (i.e., $\mathfrak{m}^{n}$ is integrally closed for all $n \geq 1$ ) then $\mathfrak{m}^{\ell}$ has the strong Rees property for every $\ell \geq 1$.

Proof. By [8, Theorem 3.1] we get $\operatorname{depth} G\left(\mathfrak{m}^{n}\right) \geq 2$ for all $n \gg 0$. Here $G\left(\mathfrak{m}^{n}\right)$ denotes the associated graded ring of $\mathfrak{m}^{n}$. It follows that $H_{\mathfrak{M}}^{1}(G(\mathfrak{m}))$ has finite length. Also as $\mathfrak{m}^{r}$ is integrally closed for all $r \geq 1$ we get that it is Ratliff-Rush closed. It follows that $\mathfrak{m}^{r}$ has SRP for all $r \geq 1$ by Theorem 3.2.

Example 3.8. Let $(A, \mathfrak{m})$ be a two-dimensional excellent normal local domain. If $\mathfrak{m}$ is a $p_{g}$-ideal (e.g. $A$ is a rational singularity), then $\mathfrak{m}^{\ell}$ has the strong Rees property for every $\ell \geq 1$.

On the other hand, "depth $G \geq 1$ " can be characterized by the Rees property.

Proposition 3.9. Put $G=G(\mathfrak{m})$, where $d=\operatorname{dim} G \geq 1$. Then the following conditions are equivalent:

(1) $\operatorname{depth} G \geq 1$.

(2) $\mathfrak{m}^{\ell}$ has the Rees property for every $\ell \geq 1$.

Proof. (1) $\Longrightarrow(2)$ : If $\operatorname{depth} G \geq 1$, then $\widetilde{\mathfrak{m}^{\ell}}=\mathfrak{m}^{\ell}$ and $\mathfrak{m}^{\ell}$ is $\mathfrak{m}$-full for every $\ell \geq 1$. In particular, $\mathfrak{m}^{\ell}$ has the Rees property.

$(2) \Longrightarrow(1):$ Now suppose $\operatorname{depth} G=0$. Then $H_{\mathfrak{M}}^{0}(G) \neq 0$. There exist an integer $\ell \geq 1$ and an element $z \in \mathfrak{m}^{\ell} \backslash \mathfrak{m}^{\ell+1}$ such that $0 \neq z^{*}=z+\mathfrak{m}^{\ell+1} \in$ $\left[H_{\mathfrak{M}}^{0}(G)\right]_{\ell} \cap \operatorname{Soc}\left(H_{\mathfrak{M}}^{0}(G)\right)$. Then $\mathfrak{m} z \subset \mathfrak{m}^{\ell+2}$. If we put $J=(z)+\mathfrak{m}^{\ell+1}$, then $\mathfrak{m} J=\mathfrak{m}(z)+\mathfrak{m}^{\ell+2}=\mathfrak{m}^{\ell+2}$. Hence

$$
J / \mathfrak{m} J=\left((z)+\mathfrak{m}^{\ell+1}\right) / \mathfrak{m}^{\ell+2} \supsetneq \mathfrak{m}^{\ell+1} / \mathfrak{m}^{\ell+2}
$$

and so $\mu(J)=\ell_{A}(J / \mathfrak{m} J)>\ell_{A}\left(\mathfrak{m}^{\ell+1} / \mathfrak{m}^{\ell+2}\right)=\mu\left(\mathfrak{m}^{\ell+1}\right)$. This contradicts the assumption that $\mathfrak{m}^{\ell+1}$ has the Rees property.

We now consider the case of $\operatorname{depth} G=1$. We need the following lemma.

Lemma 3.10. Suppose that $\operatorname{depth} G=1$. Assume that $J \supsetneq \mathfrak{m}^{\ell}$ so that $\mu(J)=$ $\mu\left(\mathfrak{m}^{\ell}\right)$. Then one can find elements $x \in \mathfrak{m} \backslash \mathfrak{m}^{2}$ and $y \in J \backslash\left(\mathfrak{m}^{\ell}+(x)\right)$ such that $x^{*}$ is $G$-regular and $0 \neq z^{*} \in \operatorname{Soc}\left(H_{\mathfrak{M}}^{0}(\bar{G})\right)$, where $z=\bar{y} \in A / x A$ and $z^{*}$ denotes the initial form of $z$ in $\bar{G}=G / x^{*} G$.

Proof. Take an element $x \in \mathfrak{m} \backslash \mathfrak{m}^{2}$ so that $x^{*}$ is $G$-regular and $J \not \subset \mathfrak{m}^{\ell}+(x)$; see the remark below for the exitence of $x$. Choose $y \in J \backslash\left(\mathfrak{m}^{\ell}+(x)\right)$. Then $y \in\left(\mathfrak{m}^{k}+(x)\right) \backslash\left(\mathfrak{m}^{k+1}+(x)\right)$ for some $k$ with $0 \leq k \leq \ell-1$. By assumption and Lemma 3.3, we have that $\mathfrak{m} y \subset \mathfrak{m} J=x J+\mathfrak{m}^{\ell+1}$. Let - denote the image of the surjection $A \rightarrow A / x A$ and put $z=\bar{y}$. Then $\overline{\mathfrak{m}} z \subset \bar{m}^{\ell+1} \subset \bar{m}^{k+2}$, which means that $0 \neq z^{*} \in\left[\operatorname{Soc}\left(H_{\mathfrak{M}}^{0}\left(G / x^{*} G\right)\right)\right]_{k}$. 
Remark 3.11. Suppose $\operatorname{depth} G=1$. For any ideal $J \supsetneq \mathfrak{m}^{\ell}$, one can find an element $x \in \mathfrak{m} \backslash \mathfrak{m}^{2}$ so that $x^{*}$ is $G$-regular and $J$ is not contained in $\mathfrak{m}^{\ell}+(x)$. In order to prove this, it suffices to consider the case $\ell\left(J / \mathfrak{m}^{\ell}\right)=1$, that is, $J=(f)+\mathfrak{m}^{\ell}$, where $f \notin \mathfrak{m}^{\ell}$ and $\mathfrak{m} f \subset \mathfrak{m}^{\ell}$.

If $J \subset \mathfrak{m}^{\ell}+(x)$ for such an element $x$ as above, then $f-a x \in \mathfrak{m}^{\ell}$ for some $a \in A$. As $x^{*}$ is $G$-regular (and thus $a^{*} x^{*} \neq 0$ ), we obtain the relation $a^{*} x^{*}=f^{*}$ in $G$. Let $G=k[X] / \mathfrak{a}$ and let $F$ be the inverse image of $f^{*}$ in the polynomial ring $k[X]$. As $k$ is an infinite field and $\operatorname{dim} k[X] /(\mathfrak{a}+(F)) \geq 1$, one can find a homogeneous element $X$ of degree one which does not vanish on $V(\mathfrak{a}+(F))$. The required assertion follows from here.

Hence we have the following.

Proposition 3.12. Suppose that $\operatorname{depth} G=1$. Let $x^{*} \in G_{1}$ be a nonzero divisor of $G$ and put $\bar{G}=G / x^{*} G$. If $\left[\operatorname{Soc}\left(H_{\mathfrak{M}}^{0}(\bar{G})\right]_{\ell}=0\right.$ for all $\ell<n$, then $\mathfrak{m}^{\ell}$ has the strong Rees property for all $\ell \leq n$.

Proof. First we note that $\operatorname{Soc}\left(H_{\mathfrak{M}}^{0}\left(G / x^{*} G\right)\right)$ is independent of the choice of $x$. In fact, the short exact sequence

$$
0 \longrightarrow G(-1) \stackrel{x^{*}}{\longrightarrow} G \longrightarrow \bar{G}:=G / x^{*} G \longrightarrow 0
$$

yields a short exact seuence

$$
H_{\mathfrak{M}}^{0}(G)=0 \longrightarrow H_{\mathfrak{M}}^{0}(\bar{G}) \longrightarrow H_{\mathfrak{M}}^{1}(G)(-1) \stackrel{x^{*}}{\longrightarrow} H_{\mathfrak{M}}^{1}(G) .
$$

Taking a socle, we get

$$
0 \longrightarrow \operatorname{Soc}\left(H_{\mathfrak{M}}^{0}(\bar{G})\right) \longrightarrow \operatorname{Soc}\left(H_{\mathfrak{M}}^{1}(G)\right)(-1) \stackrel{x^{*}}{\longrightarrow} \operatorname{Soc}\left(H_{\mathfrak{M}}^{1}(G)\right) .
$$

Since the last map is a zero map, $\operatorname{Soc}\left(H_{\mathfrak{M}}^{0}(\bar{G})\right) \cong \operatorname{Soc}\left(H_{\mathfrak{M}}^{1}(G)\right)(-1)$ is independent of the choice of $x$.

Now suppose that $\mathfrak{m}^{\ell}$ does not have SRP. Then we can find an ideal $J \supsetneq \mathfrak{m}^{\ell}$ so that $\mu(J)=\mu\left(\mathfrak{m}^{\ell}\right)$. By Lemma 3.10, there exist an element $x \in \mathfrak{m} \backslash \mathfrak{m}^{2}$ such that $x^{*}$ is $G$-regular and $0 \neq\left[\operatorname{Soc}\left(H_{\mathfrak{M}}^{0}\left(G / x^{*} G\right)\right]_{k}\right.$ for some $0 \leq k \leq \ell-1 \leq n-1$. This contradicts the assumption.

Proposition 3.13. When $\operatorname{depth} G=1$, there exists an $n \in \mathbb{N} \cup\{\infty\}$ such that $\mathfrak{m}^{\ell}$ has the strong Rees property if and only if $1 \leq \ell \leq n$.

In order to prove the proposition, it suffices to show the following lemma.

Lemma 3.14. Suppose that $\operatorname{depth} G=1$. If $\mathfrak{m}^{\ell}$ does not have the strong Rees property, then neither does $\mathfrak{m}^{\ell+1}$.

Proof. Since depth $G=1$, there exists an element $x \in \mathfrak{m} \backslash \mathfrak{m}^{2}$ so that $x^{*}=x+\mathfrak{m}^{2}$ is $G$-regular. In particular, $\mathfrak{m}^{\ell}$ and $\mathfrak{m}^{\ell+1}$ are $\mathfrak{m}$-full. By assumption and Lemma 3.3 . 
we can take an ideal $I \supsetneq \mathfrak{m}^{\ell}$ so that $\mathfrak{m} I=x I+\mathfrak{m}^{\ell+1}$. Put $J=x I+\mathfrak{m}^{\ell+1}$. Then

$$
\mathfrak{m} J=\mathfrak{m}\left(x I+\mathfrak{m}^{\ell+1}\right)=\mathfrak{m}(x I)+\mathfrak{m}^{\ell+2} .
$$

Moreover, we suppose that $J=\mathfrak{m}^{\ell+1}$. Then $x I \subset \mathfrak{m}^{\ell+1}$ and thus $I \subset \mathfrak{m}^{\ell+1}: x=\mathfrak{m}^{\ell}$. This contradicts the choice of $I$. Hence $J \supsetneq \mathfrak{m}^{\ell+1}$ and $\mu(J)=\mu\left(\mathfrak{m}^{\ell+1}\right)$. This implies that $\mathfrak{m}^{\ell+1}$ does not have SRP.

Example 3.15. For any $n \geq 1$, there exists a triple $(a, b, c) \in \mathbb{N}^{3}$ such that $A=$ $k\left[\left[s, t^{a}, t^{b}, t^{c}\right]\right]$ is a two-dimensional Cohen-Macaulay local domain such that $\mathfrak{m}^{\ell}$ has the strong Rees property if and only if $1 \leq \ell \leq n$.

Proof. For a given $n \geq 1$, we can choose an integer $a=10^{N}>2 n$. Set $b=a+1$ and $c=(a-1)(a+1)-a n$. Then $s, t^{a}, t^{b}, t^{c}$ is a minimal system of generators because $a b-a-b=(a-1)(a+1)-a \geq c(>b>a)$.

Since

$$
A=k[[s, x, y, z]] /\left(y z-x^{a+1-n}, x^{n} z-y^{a-1}, z^{2}-x^{a+1-2 n} y^{a-2}\right),
$$

we get

$$
G=G(\mathfrak{m}) \cong k[S, X, Y, Z] /\left(Y Z, X^{n} Z, Z^{2}, Y^{a}\right) .
$$

Then $S$ is an $G$-regular and $\operatorname{Soc}\left(H_{\mathfrak{M}}^{0}(G / S G)\right)$ is generated by $\overline{X^{n-1} Z} \in[G / S G]_{n}$. Hence $\mathfrak{m}^{\ell}$ has SRP if $1 \leq \ell \leq n$.

If we put $I=\left(x^{n-1} z\right)+\mathfrak{m}^{n+1} \supsetneq \mathfrak{m}^{n+1}$, then $x \cdot x^{n-1} z=y^{a-1} \in \mathfrak{m}^{a-1} \subseteq \mathfrak{m}^{n+2}$. Similarly, we have that $y \cdot x^{n-1} z \in \mathfrak{m}^{a} \subseteq \mathfrak{m}^{n+2}$ and $z \cdot x^{n-1} z \in \mathfrak{m}^{2 a-n-2} \subset \mathfrak{m}^{n+2}$. Hence $\mathfrak{m} I=\left(s \cdot x^{n-1} z\right)+\mathfrak{m}^{n+2}$, and this implies that $\mathfrak{m}^{n+1}$ does not have SRP.

Example 3.16. Let $A=k\left[\left[s, t^{4}, t^{5}, t^{11}\right]\right]$ and $\mathfrak{m}=\left(s, t^{4}, t^{5}, t^{11}\right)$. Then $\mathfrak{m}^{2}$ does not have strong Rees property. In fact, $\mathfrak{m}^{2}=\left(s^{2}, s t^{4}, s t^{5}, s t^{11}, t^{8}, t^{9}, t^{10}\right)$ and thus $\mu\left(\mathfrak{m}^{2}\right)=7$. If we put $I=\left(s^{2}, s t^{4}, s t^{5}, t^{8}, t^{9}, t^{10}, t^{11}\right)=\mathfrak{m}^{2}+\left(t^{11}\right)$, then $I \supsetneq \mathfrak{m}^{2}$ and $\mu(I)=7=\mu\left(\mathfrak{m}^{2}\right)$.

On the other hand, since $G \cong k[S, X, Y, Z] /\left(X Z, Y Z, Z^{2}, Y^{4}\right)$ (see e.g. [20, Section 2]), we have $\operatorname{depth} G=1$ and thus $\mathfrak{m}^{2}=\widetilde{\mathfrak{m}^{2}}$ is $\mathfrak{m}$-full. Moreover, since $t^{11} \in \overline{\mathfrak{m}^{2}} \backslash \mathfrak{m}^{2}, \mathfrak{m}^{2}$ is not integrally closed.

We can find an example of two-dimensional excellent normal local domains $(A, \mathfrak{m})$ for which $\mathfrak{m}^{2}$ does not satisfy the strong Rees property and $A / s A \cong k\left[\left[t^{4}, t^{5}, t^{11}\right]\right]$ for some nonzero divisor $s$ of $A$. See the next section.

\section{Point divisor on a Smooth Curve - An example $\mathfrak{m}^{n}$ Does not have STRONG REES PROPERTY}

In this section we treat a class of normal graded rings of dimension 2 and discuss whether $\mathfrak{m}^{n}$ has the strong Rees property in such rings. 
Definition 4.1. Let $k$ be an algebraically closed field and $C$ be a smooth connected projective curve of genus $g$ over $k$. We take a point $P \in C$ and define

$$
H=H_{C, P}=\left\{n \in \mathbb{Z} \mid h^{0}\left(C, \mathcal{O}_{C}(n P)\right)>h^{0}\left(C, \mathcal{O}_{C}((n-1) P)\right)\right\},
$$

where $h^{i}(C, \mathcal{F})=\operatorname{dim}_{k} H^{i}(C, \mathcal{F})$. It is easy to see that $H_{C, P}$ is an additive semigroup and $\mathbb{N} \backslash H_{C, P}$ has just $g$ elements.

We define

$$
R=R_{H}=R_{C, P}=\oplus_{n \geq 0} H^{0}\left(C, \mathcal{O}_{C}(n P) T^{n},\right.
$$

as a subring of $k(C)[T]$, where $H^{0}\left(C, \mathcal{O}_{C}(n P)=\left\{f \in k(C) \mid \operatorname{div}_{C}(f)+n P \geq 0\right\}\right.$. Namely, $f \in H^{0}\left(C, \mathcal{O}_{C}(n P)\right.$, if and only if $f$ has pole of order at most $n$ at $P$ and no other poles.

Then $R=R_{C, P}$ is a normal graded ring of dimension 2 as treated in [5], Chapter 5 , $\S 2$. In the following, we fix $H=H_{C, P}$ and write $A=k[H]$ and $R=R_{H}$ so that $R / T R \cong A$, where $T=1 . T \in R_{1}$.

We write $H=\left\langle n_{1}, \ldots, n_{e}\right\rangle$ if $H=\left\{\sum_{i=1}^{e} a_{i} n_{i} \mid a_{i} \in \mathbb{Z}_{\geq 0}(i=1, \ldots, e)\right\}$. In this case, we say that $H$ is generated by $e$ elements. We denote by $H_{+}$the set of positive elements of $H$ and denote $n \in r H_{+}$if $n=h_{1}+\ldots+h_{r}$ with $h_{i} \in H_{+}$ $(i=1, \ldots, r)$.

Remark 4.2. Given a semigroup $H$, sometimes there does not exist the pair $(C, P)$ such that $H_{C, P}=H$. But at least we know the existence of $(C, P)$ such that $H=H_{C, P}$ in the following cases (cf. [10, [11]);

(1) $k[H]$ is a complete intersection.

(2) $H$ is generated by 3 elements.

(3) $H$ is generated by 4 elements and $H$ is symmetric or pseudo-symmetric.

(4) $g(H) \leq 9$, where $g(H)$ is the number of positive integers not in $H$.

We summarize some property of $R=R_{C, P}$. We put $\mathfrak{m}=R_{+}$.

Proposition 4.3. Let $R=R_{C, P}$. An element of $R_{n}$ is denoted by $f T^{n}$, where $f \in k(C)$. We denote by $v(f)$ the order of the pole of $f$ at $P$. For non zero elements $f, g \in k(C), v(f g)=v(f)+v(g)$.

(1) $f T^{n} \in R_{n}$ if and only if $v(f) \leq n$ and $f$ has no other poles on $C$.

(2) Hence if $v(f)<n$, then $f T^{n} \in T^{n-v(f)} R$, because $f T^{v(f)} \in R_{v(f)}$.

(3) If $H_{C, P}=\left\langle n_{1}, \ldots, n_{e}\right\rangle$, which are minimal generating system, then there are elements $f_{1}, \ldots, f_{e} \in k(C)$ with $v\left(f_{i}\right)=n_{i}(i=1, \ldots, e)$ such that $R=k\left[T, f_{1} T^{n_{1}}, \ldots, f_{e} T^{n_{e}}\right]$.

(4) If $f T^{n} \in R_{n}$ and $v(f)=n$, then $f T^{n} \in \mathfrak{m}^{r}$ if and only if $n \in r H_{+}$.

(5) $T \in R_{1}$ is a super regular element of $R$. Namely, if $T x \in \mathfrak{m}^{r}$ for some $x \in R$, then $x \in \mathfrak{m}^{r-1}$. 
Theorem 4.4. Let $R=R_{C, P}$ and $H=H_{C, P}=\left\langle n_{1}, \ldots, n_{e}\right\rangle$. If for some $n \in$ $r H_{+}, n \notin(r-1) H_{+}, n+n_{i} \in(r+2) H_{+}$for $i=1, \ldots, e$, then $\mathfrak{m}^{n+1}$ does not have the strong Rees property.

Proof. By the assumption, there is some $n \in(r-1) H_{+}, n \notin r H_{+}$such that $n+$ $H_{+} \subset(r+1) H_{+}$. Then we can take $f \in k(C)$ so that $v(f)=n$ and $f T^{n} \in$ $R_{n}$. Since $f T^{n} \notin T R, f T^{n} \in \mathfrak{m}^{r-1}$ and $f T^{n} \notin \mathfrak{m}^{r+1}$. We put $I=\left(\mathfrak{m}^{r}, f T^{n}\right)$ and show that $\mu(I)=\mu\left(\mathfrak{m}^{r}\right)$. Now, let homogeneous minimal generators of $\mathfrak{m}$ be $\left\{T, g_{1} T^{n_{1}}, \ldots, g_{e} T^{n_{e}}\right\}$. Then among the homogeneous minimal generators of $\mathfrak{m}\left(f T^{n}\right),\left(f T^{n}\right)\left(g_{i} T^{n_{i}}\right) \in \mathfrak{m}^{r+1}$ by our assumption. Hence we can obtain minimal generating system of $I$ from that of $\mathfrak{m}^{r}$, interchanging $f T^{n}$ and $f T^{n+1}$, obtaining $\mu(I)=\mu\left(\mathfrak{m}^{r}\right)$.

Corollary 4.5. Let $R=R_{C, P}, H=H_{C, P}$ and $\mathfrak{m}=R_{+}$. Then the following conditions are equivalent:

(1) For all $n \geq 2, \mathfrak{m}^{n}$ has the strong Rees property.

(2) The associated graded ring of $k[H]$ with respect to $k[H]_{+}$is Cohen-Macaulay.

(3) The associated graded ring of $R$ with respect to $R_{+}$is Cohen-Macaulay.

Example 4.6. Let $H=\langle 4,5,11\rangle, C$ a smooth curve of genus 5 such that there is a point $P$ with $H_{C, P}=\langle 4,5,11\rangle$. We put $R=R_{C, P}$ and $\mathfrak{m}=R_{+}$. Since $11+4 \in 3 H_{+}$ and $11+5 \in 4 H_{+}$, we see that $\mathfrak{m}^{2}$ does not have the strong Rees property. In this example, we can easily see that $\mathfrak{m}^{n}$ is integrally closed for all $n \geq 2$.

Remark 4.7. For 3 generated semigroup $H=\langle a, b, c\rangle$, we know when the associated graded ring is Cohen-Macaulay (cf, [7, 12]).

\section{TAKAHASHI-DAO'S QUESTION}

Dao and Takahashi 21] gave two upper bounds of the dimension of the singularity category $\operatorname{dim} D_{\mathrm{sg}}(A)$ :

$$
\begin{aligned}
& \operatorname{dim} D_{\mathrm{sg}}(A) \leq(\mu(I)-\operatorname{dim} A+1) \ell \ell(I)-1 \\
& \operatorname{dim} D_{\mathrm{sg}}(A) \leq e(I)-1
\end{aligned}
$$

for any $\mathfrak{m}$-primary ideal $I$ contained in the sum $\mathcal{N}^{A}$ of the Noether differents of $A$. They posed the following question.

Question 5.1 (Takahashi-Dao). Let $(A, \mathfrak{m})$ be a d-dimensional Cohen-Macaulay local ring. Let $I \subset A$ be an $\mathfrak{m}$-primary (integrally closed) ideal. When does an inequality

$$
(d-1) !(\mu(I)-d+1) \cdot \ell \ell_{A}(I) \geq e(I)
$$

hold true (cf. [2])? 
The following theorem is motivated by the question as above. In fact, $(1) \Rightarrow(2)$ is due to Dao and Smirnov, and $(2) \Rightarrow(1)$ is also proved by them independently.

Theorem 5.2. Let $(A, \mathfrak{m})$ be a two-dimensional excellent normal local domain containing $k=\bar{k} \cong A / \mathfrak{m}$. Then the following conditions are equivalent:

(1) $\mathfrak{m}$ is a $p_{g}$-ideal.

(2) For every $\mathfrak{m}$-primary integrally closed ideal I,

$$
(\mu(I)-1) \cdot \ell \ell_{A}(I) \geq e(I)
$$

holds true.

(3) For any power $I=\mathfrak{m}^{\ell}$ of $\mathfrak{m}$, the inequality $(*)$ holds true.

Proof. (1) $\Longrightarrow(2)$ : We give a sketch of proof here for the sake of the completeness. Assume that $\mathfrak{m}$ is a $p_{g}$-ideal. Let $I \subset A$ be an $\mathfrak{m}$-primary integrally closed ideal. Then there exists a resolution of singularities $f: X \rightarrow \operatorname{Spec} A$ so that $I \mathcal{O}_{X}=$ $\mathcal{O}_{X}(-Z)$ for some anti-nef cycle $Z$ on $X$. By [13, Theorem 6.1], we have

$$
\mu(I)=-M Z+1
$$

where $M$ is an anti-nef cycle on $X$ so that $\mathfrak{m} \mathcal{O}_{X}=\mathcal{O}_{X}(-M)$.

Put $r=\ell(I)$, that is, $\mathfrak{m}^{r} \subset I$ and $\mathfrak{m}^{r-1} \not \subset I$. Then $\overline{\mathfrak{m}^{r}} \subset \bar{I}=I$. Thus $r M \geq Z$. Since $e(I)=-Z^{2}$, we have

$$
(\mu(I)-1) \ell_{A}(I)-e(I)=(-M Z) r+Z^{2}=-(r M-Z) Z \geq 0
$$

as required.

$(2) \Longrightarrow(3)$ : Trivial.

$(3) \Longrightarrow(1)$ : Now assume that inequalities

$$
\left(\mu\left(\overline{\mathfrak{m}^{\ell}}\right)-1\right) \cdot \ell \ell_{A}\left(\overline{\mathfrak{m}^{\ell}}\right) \geq e\left(\overline{\mathfrak{m}^{\ell}}\right)
$$

hold true for all integers $\ell \geq 1$. This shows that

$$
\left(\mu\left(\overline{\mathfrak{m}^{\ell}}\right)-1\right) \cdot \ell \geq e\left(\overline{\mathfrak{m}^{\ell}}\right)=e\left(\mathfrak{m}^{\ell}\right)=\ell^{2} \cdot e,
$$

where $e=e(\mathfrak{m})$. Hence $\mu\left(\overline{\mathfrak{m}^{\ell}}\right) \geq \ell e+1$.

In the case of $\ell=1$, we have that $\mu(\mathfrak{m})-1 \geq e(\mathfrak{m})$. On the other hand, Abhyankar's inequality implies that $\mu(\mathfrak{m})-1 \leq e(\mathfrak{m})$, and thus equality holds true. That is, $A$ has maximal embedding dimension in the sense of Sally ([19]). Then $\mu\left(\mathfrak{m}^{\ell}\right)=\ell e+1$. Moreover, since $G=G(\mathfrak{m})$ is Cohen-Macaulay ([18]), we obtain that $\mathfrak{m}^{\ell}=\widetilde{\mathfrak{m}^{\ell}}$ is $\mathfrak{m}$-full for every $\ell \geq 1$. Then $\mu\left(\overline{\mathfrak{m}^{\ell}}\right) \leq \mu\left(\mathfrak{m}^{\ell}\right)=\ell e+1$ by Rees property of $\mathfrak{m}$-full ideals. Hence $\mu\left(\overline{\mathfrak{m}^{\ell}}\right)=\mu\left(\mathfrak{m}^{\ell}\right)$. Then Corollary 3.6 yields that $\overline{\mathfrak{m}^{\ell}}=\mathfrak{m}^{\ell}$ because $G(\mathfrak{m})$ is Cohen-Macaulay in our case (Sally [18]). Therefore $\mathfrak{m}$ is a $p_{g}$-ideal by $[14$. 
Remark 5.3. We can show that $I=\mathfrak{m}^{\ell}$ satisfies the above inequality if $(A, \mathfrak{m})$ has maximal embedding dimension. Note that $\mathfrak{m}^{\ell}$ does not necessarily have the strong Rees property; see Sections 3 and 4.

It is known that the maximal ideal $\mathfrak{m}$ of any two-dimensional rational singularity is a $p_{g}$-ideal. So it is natural to ask the following question. Which ring the maximal ideal of which is a $p_{g}$-ideal? By a similar argument as in [6, Corollary 11.4], we can show the following. Notice that this gives a slight generalization of the fact that any two-dimensional rational singularity is an almost Gorenstein local ring.

Proposition 5.4. Let $(A, \mathfrak{m})$ be a two-dimensional excellent normal local domain containing an algebraically closed field. Let $K_{A}$ denote the canonical module of $A$. If $\mathfrak{m}$ is a $p_{g}$-ideal, then $A$ is an almost Gorenstein local ring in the sense of [6]. That is, there exists a short exact sequence of A-modules:

$$
0 \rightarrow A \rightarrow K_{A} \rightarrow C \rightarrow 0
$$

such that $\mathfrak{m} C=x C$ for some regular element $x$ over $C$.

Example 5.5. In the notation of Section 4, let $P \in C$ be such that $H_{C, P}=$ $\{0, g+1, g+2, \ldots\}$. Then the maximal ideal $\mathfrak{m}$ of $R_{C, P}$ is a $p_{g}$-ideal.

Proof. Take $f \in k(C)$ with $f T^{g+1} \in R$ and $v(f)=g+1$. Then putting $Q=$ $\left(T, f T^{g+1}\right)$, we see that $\mathfrak{m}^{2}=Q \mathfrak{m}$.

Introduce a valuation $w$ on $R$ such that

$$
w\left(f T^{m}\right)=(m-v(f))+\left[\frac{v(f)}{g+1}\right]
$$

To show that $\mathfrak{m}^{n}$ is integrally closed it suffices to show that $w\left(g T^{m}\right) \geq n$ if and only if $g T^{m} \in \mathfrak{m}^{n}$. If $w\left(g T^{m}\right) \geq n$ and $m-v(g)=r$, since $v(g) \geq(g+1)(n-r)$, $g T^{v(g)} \in \mathfrak{m}^{n-r}$ and then $g T^{m} \in \mathfrak{m}^{n}$.

Hence $\mathfrak{m}^{n}$ is integrally closed for all $n \geq 1$ and $\mathfrak{m}$ is a $p_{g}$-ideal by Proposition 2.11 .

In dimension 2, although Takahashi-Dao's inequality does not hold for general normal ring $A$, we have an inequality adding a constant depending on $A$. Also we have converse inequality for $e(I)$ changing $\ell \ell(A)$ to $\operatorname{ord}(I)$.

Proposition 5.6. Let $(A, \mathfrak{m})$ be an excellent normal local ring as in Theorem 5.2 and $I$ be an integrally closed ideal in $A$. Then we have the following inequalities;

(1) If we put $c=p_{g}(A)-\ell_{A}\left(H^{1}\left(X, \mathcal{O}_{X}(-M)\right)\right)$, then we have an inequality

$$
(\mu(I)-1+c) \cdot \ell \ell(I) \geq e(I) .
$$

Note that $c$ is an invariant depending only on $A$.

(2) If we change $\ell(I)$ to ord $(I)$, we have the converse inequality;

$$
e(I) \geq(\mu(I)-1) \cdot \operatorname{ord}(I) .
$$


Proof. We modify our argument in the proof of Theorem 5.2

(1) In the situation of the proof of Theorem [5.2, by [13, Theorem 6.1], we have $\mu(I) \geq-M Z+1-c$ and then the argument is the same as Theorem 5.2 .

(2) Since $I \subset \mathfrak{m}^{\operatorname{ord}(I)}$, we have $Z \geq \operatorname{ord}(I) M$ and hence $-Z^{2} \geq-\operatorname{ord}(I) M Z$ and $-M Z \geq \mu(I)-1$.

Example 5.7. Put $A=k[[X, Y, Z]] /(f)$, where $f$ is homogeneous of degree $n \geq 3$ and we assume $A$ is normal. Then $c=p_{g}(A)-\ell_{A}\left(H^{1}\left(X, \mathcal{O}_{X}(-M)\right)\right)=\left(\begin{array}{c}n-1 \\ 2\end{array}\right)$ in this case. In fact, if we take $I=\mathfrak{m}^{s}$ with $s \geq n$, then we have $\ell \ell(I)=s, \mu(I)=$ $s n-(n-3) n / 2$ and we see that $c=\left(\begin{array}{c}n-1 \\ 2\end{array}\right)$ is best possible.

In dimension $\geq 3$, we can take $A$ so that there is no constant $c$ for which the inequality

$$
(d-1) !(\mu(I)-d+1+c) \cdot \ell \ell(I) \geq e(I)
$$

hold for all integrally closed ideal $I$. See the next example.

Example 5.8. Let $A=k[[x, y, z, w]] /(f)$, where $f$ is a homogeneous polynomial of degree $n$ and we assume $A$ is normal. Then we can see if $n \geq 5$, putting $I=\mathfrak{m}^{s}$, to have inequality $(d-1) !(\mu(I)-d+1+c) \cdot \ell \ell(I) \geq e(I)$ holds we have to take $c$ arbitrary large when $s$ tends to infinity.

\section{What Ideals HaVe SRP ?}

We have shown under certain condition, $\mathfrak{m}^{n}$ has SRP and also in regular local rings of dimension 2, $\mathfrak{m}^{n}(n \geq 1)$ are only ideals with SRP in Example 2.9. In this section, we ask if that this property characterize regular local rings and some Veronese subrings in dimension 2. We get a partial result for rational singularities.

Proposition 6.1. Let $(A, \mathfrak{m})$ be a two-dimensional rational singularity and assume that $A / \mathfrak{m}$ is algebraically closed. Then we have the following results concerning the strong Rees property for integral closed ideals.

(1) If an integrally closed ideal I has the strong Rees property, then I is a good ideal in the sense of [4].

(2) If the minimal resolution of $\operatorname{Spec}(A)$ has more than two exceptional curves, then there are integrally closed ideals $I \neq \mathfrak{m}^{n}$ with the property such that $\mu\left(I^{\prime}\right)<\mu(I)$ for every integrally closed ideal $I^{\prime}$ strictly containing $I$.

Proof. If $A$ is a rational singularity, and if $I=I_{Z}$ as in the proof of Theorem 5.2, we have shown $\mu(I)=-M Z+1$.

(1) If $I$ is not good and $I^{\prime}$ is the minimal good ideal containing $I^{\prime}$, we have $\mu(I)=\mu\left(I^{\prime}\right)$. Hence $I$ does not have SRP. (2) If $I=I_{Z}$ has SRP, then $Z$ is defined on the minimal resolution of $\operatorname{Spec}(A)$ and the property " $\mu\left(I^{\prime}\right)<\mu(I)$ for every integrally closed ideal $I^{\prime}$ strictly containing $I$ " is equivalent to say that "for every cycle $Z^{\prime}<Z,-M Z^{\prime}<-M Z$ or $M\left(Z^{\prime}-Z\right)>0$. If the minimal resolution of 
$\operatorname{Spec}(A)$ contains more than two curves, we can construct such $Z \neq n M$ with that property.

Conjecture 6.2. We believe that if $(A, \mathfrak{m})$ is a local ring of dimension $d \geq 2$ and if $\mathfrak{m}^{n}$ are only ideals of $A$ which has the strong Rees property, then $\operatorname{dim} A=2$ and either $A$ is a regular local ring or $\hat{A} \cong k\left[\left[X^{r}, X^{r-1} Y, \ldots, Y^{r}\right]\right]$ for some $r$.

Acknowledgment 1. The authors would like to thank Hailong Dao for giving us a motivation of this work. Moreover, they would like to thank Shiro Goto, Jürgen Herzog, Ryo Takahashi, Junzo Watanabe and Santiago Zarzuela for giving us several valuable comments.

The second author is supported by Grant-in-Aid for Scientific Research (C) 26400053. The third author is supported by Grant-in-Aid for Scientific Research

(C) 16K05110.

\section{REFERENCES}

[1] J. Asadollahi and T. J. Puthenpurakal, An analogue of a theorem due to Levin and Vasconcelos, Commutative algebra and algebraic geometry, 9-15, Contemp. Math., 390, Amer. Math. Soc., Providence, RI, 2005.

[2] H. Dao and I. Smirnov, The multiplicity and the number of generators of an integrally closed ideal, arXiv:1703.09427

[3] S. Goto, Integral closedness of complete intersection ideals, J. Algebra 108 (1987), 151-160.

[4] S. Goto, S. Iai, and K.-i. Watanabe, Good ideals in Gorenstein local rings, Trans. Amer. Math. Soc. 353 (2001), no. 6, 2309-2346 (electronic).

[5] S. Goto and K. -i.Watanabe, On graded rings, I, J. Math. Soc. Japan, 30 (1978), 179-213.

[6] S. Goto, R. Takahashi and N. Taniguchi, Almost Gorenstein rings-towards a theory of higher dimension, J. Pure Appl. Algebra 219 (2015), 2666-2712.

[7] J. Herzog, When is a regular sequence super regular?, Nagoya Math. J. 83 (1981), 183-195.

[8] S. Huckaba and C. Huneke, Normal ideals in regular rings, J. Reine Angew. Math. 510 (1999), 63-82.

[9] S. Itoh, Coefficients of normal Hilbert polynomials, J. Algebra 150 (1992), no.1, 101-117.

[10] J. Komeda, On the existence of weierstrass points with a certain semigroup generated by 4 elements, Tsukuba J. Math. 6 (1982), no.2, 237-270.

[11] J. Komeda, and A. Ohbuchi, Existence of the non-primitive Weierstrass gap sequences on curves of genus 8, Bull Braz Math. Soc. News Series 39 (2008), 109-121.

[12] L. Robbiano and G. Valla, On the equations defining tangent cones, Math. Proc. Camb. Phil. Soc. 88 (1980), 281-297.

[13] T. Okuma, K.-i. Watanabe and K. Yoshida, Good ideals and $p_{g}$-ideals in two-dimensional normal singularities, manuscripta math. 150 (2016), 499-520.

[14] T. Okuma, K.-i. Watanabe and K. Yoshida, Rees algebras and $p_{g}$-ideals in a two-dimensional normal local domain, Proc. Amer. Math. Soc. 145 (2017), no.1, 39-47.

[15] T. J. Puthenpurakal, Ratliff-Rush filtration, regularity and depth of higher associated graded modules. I, J. Pure Appl. Algebra 208 (2007), no.1, 159-176.

[16] T. J. Puthenpurakal, Ratliff-Rush filtration, regularity and depth of higher associated graded modules. Part II, J. Pure Appl. Algebra 221 (2017), no. 3, 611-631.

[17] L. J. Ratliff, Jr. and D. E.Rush, Two Notes on Reduction of ideals, Indiana Univ. Math. J. 27 (1978), no.6, 929-934. 
[18] J.D. Sally, On the associated graded ring of a local Cohen-Macaulay ring, J. Math. Kyoto Univ. 17 (1977), 19-21.

[19] J.D. Sally, Cohen-Macaulay local rings of maximal embedding dimension, J. Algebra 56 (1979), 168-183.

[20] J.D. Sally, Cohen-Macaulay Local Rings of Embedding Dimension $e+d-2$, J. Algebra 83 (1983), 393-408.

[21] H. Dao and R. Takahashi, Upper bounds for dimensions of singularity categories, C. R. Acad. Sci. Paris, Ser. I, 353 (2015), 297-301.

[22] J. Watanabe, m-full ideals, Nagoya Math. J. 106 (1987), 101-111.

[23] K.-i. Watanabe, Chains of integrally closed ideals, Commutative algebra (Grenoble/Lyon, 2001), 353-358, Contemp. Math., 331, Amer. Math. Soc., Providence, RI, 2003.

Tony J.Puthenpurakal, Department of Mathematics, IIt Bombay, Powai, Mumbai 400076, INDIA, EMAIL: TPUTHEN@GMAIL.COM

Kei-ichi Watanabe, Department of Mathematics, College of Humanity and Sciences, Nihon University, Setagaya-Ku, TOKYo, 156-8550, JAPAn, Email: WATANaBe@Math.Chs.NihonU.AC.JP

Ken-ichi Yoshida, Department of Mathematics, College of Humanities and Sciences, Nihon University, Setagaya-Ku, Tokyo, 156-8550, JAPAn, Email: yoshida@Math.Chs.NihonU.AC.JP 